\title{
THE GYNECOLOGIC SIGNIFICANCE OF APPENDICITIS IN EARLY LIFE
}

\author{
WILLIAM P. GRAVES, M.D. \\ BOSTON
}

An article dealing with the relationship between appendicitis and pelvic disease may seem like a profitless reversion to ancient gynecologic history; nevertheless, in the consideration of even so wellworn a subject as this, fresh experience adds new points of view, and the recording of such experience cannot be without some value.

Fifteen and twenty years ago, the output of literature relating to the appendix and the female pelvis was colossal. It was, however, devoted mainly to the discussion, at that time absorbingly important, of the routine removal of the appendix during gynecologic operations, Overwhelming evidence was produced showing not only that the appendix is frequently involved secondarily in pelvic inflammatory disease, but that unsuspected inflammations of the appendix, often unrecognizable by gross inspection, may occur entirely independently of pelvic lesions. Many writers, such as Fraenkel, Pollak and Bandler, recognized also the possibility of an inflammatory process traveling from the appendix as a primary focus to the pelvic organs. This aspect of the discussion, however, played only a minor rôle. Some even denied the existence of pelvic inflammation as the result of appendicitis; others regarded it as being so infrequent as to be of little practical significance. Thus, Hunter Robb as late as 1905 in an article read before the American Gynecological Society failed to "find in 1,000 laparotomies that the appendix had been the primary seat of the disease (pelvic inflammation)." Child, in the most important contribution on the subject in recent years (1919), makes the following statement: "Where the primary infection is in the appendix, it is unusual to observe any very extensive pathologic changes in the pelvic organs; a few restricting adhesions that aside from the subjective symptoms they may cause by restricting the mobility of these organs are of little moment, seldom if ever causing permanent tissue changes to be observed."

The campaign carried on for the routine removal of the appendix during pelvic operations was rapidly won, through the influence chiefly of American surgeons under the leadership of such intrepid operators as Deaver, Kelly, Peterson and others, so that by the year 1905 the routine removal of the appendix, whether diseased or not, became, excepting for special contraindications, a well-nigh universal custom. 
With this question settled, the topic of the interrelationship between the appendix and the pelvic organs lost its interest, so that within the last fifteen years, the literature has been much less voluminous.

It is the object of this article to review the subject with especial reference to that neglected aspect of it which is represented by the descending infections from the appendix as a primary focus. My attention has many times been drawn to this condition by encountering extensive adhesions in the pelves of women who had suffered from early appendicitis and in whom the chance of a gonorrheal or a puerperal infection could by all possible moral certainty be excluded. The idea of writing an article on the subject has been stimulated by several recent conversations with experienced surgeons, some of whom, to my surprise, have either expressed an entire ignorance of the condition or characterized it as of small importance.

\section{MEANS OF COMMUNICATION OF INFECTION}

In order to clarify the special points which $I$ wish to make, it is necessary to review the commonly accepted theories regarding the means of communication by which infection may pass between the appendix and the uterine adnexa. In the earlier literature, the so-called appendiculo-ovarian ligament, or ligament of Clado, was regarded as the chief medium of infectious communication. This structure was described by Clado as a peritoneal fold of the posterior abdominal wall, more or less definitely marked, springing from the infundibulopelvic ligament, and passing upward to the cecal fossa to a termination at the base of the appendix. It was said to contain the spermatic vein and artery, the spermatic nerves, and numerous lymph vessels from the right ovary and the uterine body. Great clinical importance was assigned to this ligament by such early writers as Broschke, Weldeyer, Nagel, A. Martin and Doederlein; but in the light of later observations less significance was attached to it. Barnsby (1898) was unable to find it in 127 necropsies; Füth in the same year could identify it only exceptionally. Other investigators, both surgeons and anatomists, found it inconstant. Moreover, experimentation by injecting the blood and lymph vessels failed to substantiate the claim of Clado of a specific vascular and lymphatic connection between the appendix and ovary. In 1902, I published a short article demonstrating the appendiculo-ovarian ligament in a fetus of 5 months, but in the course of a long surgical practice it has been encountered as a well-defined ligament comparatively rarely. I am inclined to regard the structure as a fetal rest, possibly a relic of the upper portion of the mesorchium, ${ }^{1}$

1. This is denied by Professor Fawcett (Bristol). 
or it may even be merely an accidental prolongation of the infundibulopelvic ligament (Paterson). We are justified in concluding, therefore, that Clado's ligament exerts at the most only an incidental influence in conducting infection to or from the pelvis.

The various methods of infectious communication have been thus described: intraperitoneally, (1) by the blood and lymph vessels of Clado's ligament; (2) through the new vessels formed in adhesions to the appendix or adnexa, or (3) along the adherent intestine, or (4) by direct contact of the appendix and adnexa: extraperitoneally (1) through the subserous cellular tissue of Clado's ligament, or (2) through that portion of the general pelvic cellular tissue which extends from the parametrium and broad ligament to the paracecum. An additional method, rarely mentioned by the earlier writers, is the descent by gravity of products of inflammation from the appendix into the pelvis. In considering these various methods, those relating to Clado's ligament may safely be disregarded. Those relating to extension through preformed adhesions may also be discarded for this immediately begs the question of how the adhesions were originally formed. We have left for consideration then the methods of communication represented by contact, gravity and extension through the subperitoneal cellular tissue. A phlegmonous infection involving the cellular tissue from the paracecum to the parametrium is quite possible. Authentic cases have been reported of parametritis following acute appendicitis during pregnancy. It is conceivable that the pelvic abscesses that occasionally appear after an operation for acute appendicitis, may have originated by extension through the cellular tissue, though it is probable, as Hermes has pointed out, that in the great majority of cases the infection extends intraperitoneally, that is to say, by gravity or contact. Higgins (in 1913) described in a male child of $31 / 2$ years an extensive cellulitis starting from an acute appendicitis and terminating in a perforating pararectal abscess. I have not been able to find authentic evidence of a distal secondary infection of the appendix from an initial parametritis. In other words, when an associated inflammatory process between appendix and adnexa does occur through the cellular tissue, its progress appears to have been descending rather than ascending. It is, of course, possible that when a severe sactosalpingitis has invaded the pelvic cellular tissue, the deep cellulitis may extend as far as the appendix and cecum, especially if they have a low attachment. In my experience, the high involvement of the cellular tissue is far more common on the left side, often with serious implication, and even obstruction of the sigmoid.

Thus we are forced to conclude that, whereas a primarily affected appendix may transmit its infectious process to the pelvis in three 
different ways, that is, by contact, gravity and through the agency of the subperitoneal cellular tissue, inflammations of the pelvic organs involve the appendix secondarily only by contact. And this statement applies whether we are dealing with gonorrheal salpingitis, puerperal peritonitis or with neoplastic tumors of the uterus and its adnexa. It is pertinent probably for the most part to genital tuberculosis.

Such a conclusion reached more or less by reasoning is substantiated by personal experience, for excepting in cases in which the appendix is actually implicated in the adherent mass of a pelvic inflammation or is attached to a pelvic tumor in the manner of any mobile organ of the peritoneal cavity, I have never observed that the appendix exhibits a much greater tendency to disease than is apparent in association with noninflammatory pelvic conditions.

It is to be noted that appendicitis secondary to inflammation of the adnexa, unless the implicating process is very destructive, is of the nature of a periappendicitis. It is also probable that an appendix involved in adhesions is more susceptible to infection and ulceration of its mucous membrane, so that, theoretically at least, an essential idiopathic appendicitis may result indirectly from pelvic disease. Such an appendix may return the compliment and reinfect the originally offending organ with disastrous results. An example of this kind of interchange may be seen in cases of purulent ovarian cystomas, the contents of which have been inoculated by an adherent perforating appendix. Intestinal reinfections are, however, not confined to the appendix for they may, of course, proceed from any loop of adherent bowel, and indeed are very common in secondary sigmoiditis.

On the other hand, infectious processes transmitted to the pelvis from a primary appendicitis by gravity or contiguity are essentially peritonitic in character, so that a secondary salpingitis, for example, would ordinarily be of the nature of a perisalpingitis. On account of the open ostium of the tube, however, it seems evident that when the adnexa are swimming in the pus of an appendicular pelvic abscess, the lumen of the tube may be invaded, and a typical sactosalpinx may result (Bandler). McNaughton-Jones reports two such cases in unmatured girls, aged 10 and 11 years, respectively.

Although we have shown that a descending infection from the appendix to the pelvis is more readily effected than an ascending infection from the tube, it must not be inferred that when both organs are involved the appendicitis is more commonly primary. In fact, quite the reverse is true. Acute pelvic inflammation from all causes is more frequent than acute exudative appendicitis. When one takes into account the fact that in 25 per cent. of all women the appendix lies in the pelvis (Kelly), the chances of a secondary involvement of the 
appendix are very great even though we eliminate all the theoretical methods of infectious access excepting that of contiguity. It is obvious that in many cases of appendiculosalpingitis, it is impossible to make a definite diagnosis as to the primary seat of infection, even by the microscope, and for that reason it would be difficult to secure trustworthy comparative figures. Although such a comparison, if it could accurately be made, would show a great preponderance of ascending infections, it would also reveal the fact that pelvic adhesive peritonitis as a result of primary appendicitis is much more frequent than is commonly supposed. It is necessary to protest against the teaching that nontuberculous inflammatory processes encountered in the pelvic organs are practically all due to gonorrhea or puerperal sepsis.

There is no doubt that the secondary pelvic inflammations are more common and more serious when they follow appendicitis in the young. In the immature, the appendix, even in its normal position, lies in fairly close proximity to the right tube, while a low hanging appendix may even rest in the posterior culdesac. Moreover, the bony pelvis of the young is straighter, that is to say, less canting, than one that is fully developed, so that the influence of gravity acts more directly on a free exudate from above. Hence in the young the anatomic conditions are less favorable for a local walling off of an appendix abscess than in adult women in whom there is greater distance and a less direct course between the appendix and the adnexa.

The appended case reports illustrate well the immediate and late changes in the pelvic organs, which may result as a consequence of youthful acute appendicitis.

EFFECT OF CHRONIC APPENDICITIS ON THE PELVIC ORGANS

It remains now to inquire whether secondary changes may be effected in the pelvic organs by a chronic appendicitis. The solution of this problem offers greater difficulties inasmuch as the histologic data of a given case are less well-defined, and it is necessary to resort in part to speculation.

It seems to be well established that the appendix may undergo considerable grades of inflammation and yet eventually be restored to a condition of approximate normality. It is entirely probable that during the inflammatory periods of a chronic appendicitis, a serous, or serofibrinous exudate is produced which finds its way by gravity into the true pelvis. One often encounters such an exudate in apparently uncomplicated pelves and wonders whence it came. It is quite within reason to suppose that, although this exudate is usually absorbed by the peritoneum, under certain conditions it may be sufficient either through bacterial or chemical influence to destroy the superficial epi- 
thelium of the pelvic peritoneum and to stimulate the subserous connective tissue into the formation of organized plastic adhesions; or it may itself become organized and form the basis of adhesions.

In this way may be explained theoretically the cases not infrequently encountered, in which without sign or history of gonorrhea or puerperal sepsis, adhesions are found in the posterior culdesac, or implicating the surfaces of the adnexa while the appendix shows only mild evidence of disease, or perhaps none at all, to gross appearance. If besides the pelvic adhesions there is added a well-defined chronic appendicitis, or the scar of an appendix operation performed in youth, our explanation becomes still more plausible; certainly more so than the attempt to ascribe the condition to an entirely improbable gonorrheal infection.

The pathologic tissue changes that take place later in a secondary pelvic inflammation depend on the severity of the initial appendicular infection. In cases in which there has been an overwhelming flood of pus in the pelvis, the resulting adhesive peritonitis may be so extreme as to involve completely the uterus and both adnexa. The immature uterus becomes fixed, usually in the position of retrocessed anteflexion, and is prevented from attaining its full development. The ovaries buried and immobilized are likely to retain their infantile character of form, usually cystic, with a white, thickened, unscarred, albuginea; or they may develop retention cysts of moderate size. The tubes, though encased in adhesions, may show remarkably little destructive change. It is sometimes found that after being dissected free, the ostia though inverted are only delicately adherent and that by careful manipulation the fimbriae may be made to roll out into their normal efflorescent form, an important practical point to remember when operating for sterility.

The severe cases of this kind that I have encountered in my practice (in which gonorrhea could with certainly be ruled out) have been observed during an operation, usually for sterility, perhaps years after the attack of appendicitis in early life. In some of the most adherent cases there has been in the patient's history a remarkable absence or mildness of pelvic symptoms. In several instances, when bimanual examination even under an anesthetic has revealed nothing more than an infantile uterus, the pelvic organs have proved to be so buried in adhesions as to be almost indistinguishable. It is as if there had been a complete organization of adhesions and adjustment of internal genitalia during the period of development and growth. On more than one occasion, I have felt after releasing the adhesions that it would have been better to leave the pelvis entirely alone. 
The adherent organs in a severe case are extremely difficult to -separate, but there is not that stiffness and hardness seen in adhesive tuberculosis, nor the tissue destruction of an old gonorrheal process.

From the cases of universal adherence, there are all grades of secondary plastic pelvic peritonitis to cases in which there is only a mild posterior perimetritis or a few insignificant adhesions of the right adnexa.

One of the most serious consequences of appendicitis in early life with pelvic involvement is sterility, and it has been in the attempt to remedy this complaint that I have most often encountered the condition. Theoretically, conservative operations for the relief of sterility in cases of secondary pelvic inflammation should be more successful than in cases following gonorrheal salpingitis, for there is not the same degree of tissue destruction of the tubes and ovaries. Practically, however, the outlook for a cure of sterility is not very good, at least it has been so in my experience, even when the tubes and ovaries can be left in a comparatively normal condition. On the other hand, I have observed that the symptomatic results of a conservative operation for pelvic adhesions secondary to appendicitis are much better than after a similar operation for primary gonorrheal adhesions. This is to be expected, for there is not the same tendency in the former to a reorganization of adhesions, nor is there the possibility of leaving behind in the conserved tissues, latent sources of the original infection.

Much has been written regarding early appendicitis, as a causative factor in a later ectopic pregnancy. Numerous cases have been reported, in which the anatomic relationship of an ectopic pregnancy and an acute or chronic appendicitis, make it extremely probable that in this or that particular instance there was a causal connection between the two lesions. Theoretically, it is conceivable that the serous exudate which, as I have heretofore stated may sink into the pelvis during the exacerbations of a chronic appendicitis, may create minor tissue changes in the tubes sufficient to result in an ectopic pregnancy. It is probable that only a minute abnormality in the tube may retard the normal progress of the impregnated ovum long enough to cause it to nidate in the tubal mucous membrane. The plastic influence of a descending pelvic exudate may be exerted on either or both tubes, but it may be expected that a chronic appendicitis would more frequently affect the right tube. In a series of forty-seven cases of ectopic pregnancy, I found the condition only slightly more frequent on the right than on the left. On the other hand, in the right-sided cases the appendix was found diseased in 80 per cent. whereas in the left-sided cases it was diseased in only 57 per cent. These figures, though comprising 
too small a number of cases, are at least suggestive that appendicitis must be reckoned with as an important factor in extra-uterine. pregnancy.

The relationship of early appendicitis to dysmenorrhea is a subject to which gynecologists have given considerable attention. It is a matter of constant observation that the appendicular colic of a chronic appendicitis is usually more marked at the menstrual period, and frequently is experienced only at that time. The same may be said of the pains of a plastic adnexitis, whether it be from a tubal or appendical origin. Such symptoms are due to the effect of the general catamenial congestion on adherent organs, and must be grouped under the heading of secondary dysmenorrheas.

The relationship of appendicitis to essential dysmenorrhea is a more difficult and as yet an unsolved problem. No gynecologist can have failed to note the frequency with which young patients with essential dysmenorrhea complain also of appendicular pain during or between the periods. Most of these patients have an abnormality of uterine position, most commonly in the form of retrocessed hyperanteflexion. Genital hypoplasia is frequent. There is in the majority of cases no secondary inflammatory reaction in the pelvis. In a series of 100 cases, taken from hospital records, in which operation was performed for essential dysmenorrhea, I find that the appendix was diseased in 63 per cent. This series is to be contrasted with one of 100 noninflammatory pelvic conditions, cases of essential dysmenorrhea being excluded, in which the appendix was diseased in only 33 per cent.; and also with a series of cases of pelvic inflammation in which the appendix was abnormal in 47 per cent.

We are confronted then with the indubitable fact that the genital abnormalities that produce symptoms of essential dysmenorrhea are associated with an unusually high percentage of diseased appendices. Is there any causal relationship between the two conditions, and if so, in what way is it effected? This question has not as yet been satisfactorily answered. Lapeyre, in 1913, rather vaguely explained it on the ground of correlated sclerosis of the appendix and ovaries, but did not answer the main question. It has been a matter of personal observation that essential dysmenorrhea is conditioned rather on the position and tissue-consistency of the uterus than on functional or anatomic changes in the ovaries. In addition to the hypoplasia and retrocessed hyperanteflexion of the uterus heretofore mentioned, one frequently encounters short, thickened, uterosacral ligaments often tender to the touch, and suggesting a chronic posterior (sometimes called superior) parametritis. In the more obstinate cases there is also often found a cicatricial band at the internal os, giving the impression 
of a partial stricture. Is it not possible that these metrial and parametrial cicatrices are the rests of some early changes secondary to an inflammatory process in the intestinal track? If we accept the theory that Jackson's membranes, Lane's kinks, folds of Treves, attic adhesions, and the numerous other organized intestinal bands are the result of early, even fetal, peritonitic processes, it is not difficult to imagine that such inflammations may be imparted to the pelvic organs through the channel of the subserous cellular tissue, and there leave traces which may later limit the full development of such organs. Perhaps in this way may be explained the amazing frequency of genital hypoplasia in women. Perhaps too the colic and "growing" pains of young children are in reality peritonitic in character and are often the ultimate cause of restricted genital development.

The foregoing speculation as to the effect of fetal adhesions is not without some scientific support. I have observed a case of uterus didelphys caused by an intervening adhesive band between the bladder and rectum, which prevented the complete union of Müller's ducts. Other similar cases have been reported.

A thorough realization of the pathology of early appendicitis in the female emphasizes several very practical lessons, which may be summarized thus :

Appendicitis in childhood and young girlhood is an affection which must be regarded not simply with reference to the diseased appendix itself but to the serious harm which it may exert on the pelvic organs, if left to work out its own destiny in a state of chronic inflammation. Early operation is therefore indicated in children when there is any suspicious evidence of appendicular infection. In the acute stage, the appendix should be removed immediately to forestall if possible a secondary involvement of the adnexa. If pus is present, every effort should be made to drain the pelvis, it being feasible in certain cases to drain the pouch of Douglas through the vagina. Excepting in cases of localized abscess it is advisable to make a median line incision in order that the pelvic organs may be inspected, and that any abnormalities of position or plastic adherence may be remedied by a proper surgical procedure.

\section{REPORT OF ILLUSTRATIVE CASES}

The three cases herewith reported are illustrative of the serious results that may follow early appendicitis.

CASE 1.-History.-M. M., aged 14, had an abdominal attack in 1915, which was diagnosed by a physician as acute salpingitis with sactosalpinx. Expectant treatment was advised. The condition grew worse and on the third day after the attack demanded immediate intervention.

Examination.-The patient was well developed, puberty was established and the hymen was unruptured. The condition was diagnosed as acute appendicitis with pelvic abscess. 
Operation and Result.-An operation, at which I assisted, was performed under disadvantageous conditions in a country town. A right rectus incision was made, and the pelvis and abdomen were found filled with pus. The right tube which appeared first in the wound was very large and red. The appendix, which was located with some difficulty, was gangrenous and perforated. It was removed, and the pelvis was drained. There was a slow, stormy convalescence resulting in recovery.

Five years after the operation, the patient was seen, but no examination was made. She had suffered considerable pelvic pain since the operation. The menses, which were well established at the time of operation, have been somewhat irregular and scanty since, and there has been severe dysmenorrhea. Though unusually well developed at $\mathbf{1 4}$, she has grown little since, presenting the general appearance of incomplete development.

CASE 2.-History.-Mrs. R. P. K., aged 27, who had been married for five years and who had no children, complained chiefly of sterility. Menstruation was very irregular, the flow was profuse and clotted. Otherwise the patient was perfectly well. She had severe appendicitis at about the age of puberty for which an operation was performed.

Examination.-The patient was well developed and tended to be fat. There was retroversioflexion of the uterus. Both ovaries were prolapsed and the uterus was undeveloped. There was no possibility of gonorrhea or puerperal sepsis.

Operation and Result.-Operation was performed, Jan. 21, 1911. The pelvis was filled with adhesions extending from an old appendicitis scar. These were released. The tubes when released appeared normal; both ovaries showed a white dense albuginea. The left ovary contained a small fibroma. No corpus luteum was found in either ovary and no scarring. Both ovaries were very large. They were resected to normal size, and the uterus was restored to normal position by a modified Gilliam's operation. Convalescence was uneventful. Although the patient was perfectly well after the operation, fertility was not restored. A similar operation was performed later by another surgeon with similar results.

CASE 3.-History.-Mrs. H. B., aged 30, who had been married five years, complained chiefly of sterility. She felt perfectly well. Menstruation had been regular, but there had been some dysmenorrhea. There was no possibility of gonorrhea or puerperal sepsis. She had acute appendicitis during her girlhood for which operation was performed.

Examination.-The patient was well developed. The uterus was anteflexed and retrocessed. The left ovary was prolapsed and somewhat tender.

Operation and Result.-Operation was performed, May 22, 1916. The pelvic organs were found completely buried in adhesions which extended from an old appendicitis scar. The pelvic organs were released from the adhesions with the greatest difficulty. The left ovary contained a blood cyst. The left tube and ovary were so damaged that they required resection. The release of the adhesions left a bleeding area in the pelvis requiring ligature of a branch of the left uterine vein and packing to control venous oozing. The patient had a stormy convalescence, but recovered eventually. She has been perfectly well since, although fertility has not been restored. There has been no disturbance of the menses.

244 Marlboro Street. 